\title{
Novel image detector of fixed-valued impulse noise with high noise intensity
}

\author{
Haijiang Hu, Shaojing Song* and Weifei Gan \\ College of Engineering, Shanghai Polytechnic University, 201209, Shanghai, China
}

\begin{abstract}
Keywords: Impulse noise, Salt\&Pepper noise, Noise detector, Slant angle, noise intensity.

Abstract. Impulse noise is a common noise in digital image processing, which includes the fixed-valued impulse noise and the random-valued impulse noise. A novel method is proposed to detect the fix-value impulse that uses the deviation of slant angle as test object to recognize whether a noise candidate is impulse noise pixel. Distinguishing the average method and medium method, the method uses the new test object to detect the impulse noise. The mathematical model and the detection algorithm of this method are stated and analyzed, and the experiments show the performance of this method under the different circumstance. The biggest advantage of this method is to detect the fixed-valued impulse noise correctly and robustly under the different noise intensity that is up to 0.95 .
\end{abstract}

\section{Introduction}

In the digital image processing [1], one of the key problems is the detection and filtering of noise. Two noise models are often used to analyze the image noise: additive Gaussian noise and impulse noise. The additive Gaussian noise is defined by adding a value with a zero-mean Gaussian distribution to every original image pixel. Impulse noise is another kind of common noise in digital image, which includes fixed-valued impulse noise and randomvalued impulse noise. For the fixed-valued impulse noise, also called Salt\&Pepper noise, the value of polluted pixel is equal to minimum or maximum of pixel value according to the same probability. Many methods are proposed in the recent years, such as TSM filter [2], MSM filter [3], SBF [4], ROR-NLM [5], SAWM filter [6], and ABDND [7]. In this paper, a new method is proposed to detect the fixed-valued impulse noise, which is effective to detect heavy polluted image especially. Instead of average detection and medium detection, the deviation of slant angle is chosen as a new test object for the noise detection. Therefore we call the new detector as DSA (deviation of slant angle).

\footnotetext{
*Corresponding author: hjhu@sspu.edu.cn
} 


\section{Definition of slant angle}

We introduce the noise model simply because many related papers have already described the details of impulse noise. If an image is polluted by the impulse noise, some original pixels are replaced by the noise pixels. If xij and yij are defined as the pixel value of original image and polluted image at the location $(i, j)$, respectively, we define

$$
y_{i j}=\left\{\begin{array}{ll}
x_{i j}, & \text { with probablity } 1-p \\
n_{i j}, & \text { with probablity } p
\end{array},\right.
$$

where $\mathrm{p}$ is impulse noise ratio, nij is the noise value at the location $(\mathrm{i}, \mathrm{j})$ that is independent from xij, respectively. If nij only equals to the minimum or maximum of pixel value, the noise is called the fixed-valued impulse noise or Salt\&Pepper noise. If the noise intensity is low, many detection methods can achieve excellent performance. However, if the noise intensity is very high, such as up to 0.95 , it is difficult for most of methods to distinguish between the uncontaminated pixel of which value equals to 0 or 255 and the noise pixel very well.

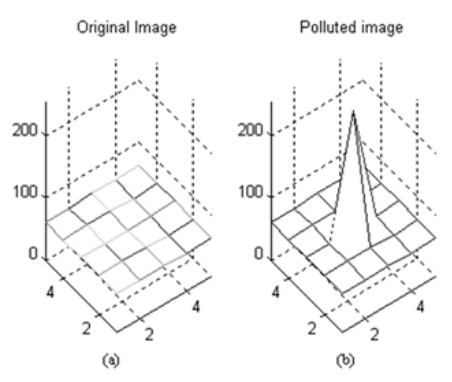

Fig. 1. Simplest polluted scheme with one polluted pixel. (a) Original image. (b) Polluted image.

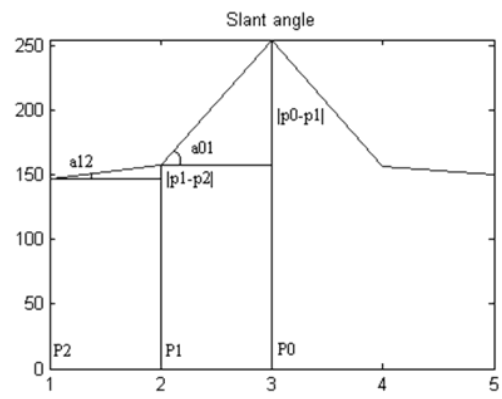

Fig. 2. Definition of slant angle.

In the simplest scene, the center pixel is polluted in a $5^{*} 5$ area while other pixels in this area are normal, which is shown in Fig.1. We can find the left one looks like the plain while the right one looks like the peak in the plain. So the slant angle is a new feature for the fixedvalued impulse noise detection. In Fig.2, P0, P1, and P2 are defined as the center pixel, the neighbor of $\mathrm{P} 0$, the edge pixel in a $5 * 5$ area, respectively. Therefore the slant angles a01 in Fig.3 is defined as Eq.2.

$$
a_{01}=\arctan \frac{p_{0}-p_{1}}{D_{p 0 p 1}} .
$$


In Eqs.2, we define a01 as the slang angel between $\mathrm{P}_{0}$ and $\mathrm{P}_{1}, D$ as the distance between $\mathrm{P}_{0}$ and $\mathrm{P}_{1}$, $\mathrm{p}_{0}$ is the value of pixel $\mathrm{P}_{0}, \mathrm{p}_{1} \mathrm{I}$ the value of pixel $\mathrm{P}_{1}$, respectively. Obviously, the value range of slant angle is $\left(-\frac{\pi}{2}, \frac{\pi}{2}\right)$. The same to Eq.2, we can also calculate the slant angel between $\mathrm{P}_{1}$ and $\mathrm{P}_{2}$ as follows:

$$
a_{12}=\arctan \frac{p_{1}-p_{2}}{D_{p 1 p 2}}
$$

\section{Deviation of slant angle}

From Fig.1 and Fig.2, we know intuitively the probability that P0 is a noise pixel is high if the difference between $\mathrm{a} 01$ and a12 is large. So the deviation of slant angle can be used for the noise detection. We define the deviation of slant angle in the route P0-P1-P2 as follows:

$$
D e v=\left|\arctan \frac{p_{0}-p_{1}}{D_{p 0 p 1}}-\arctan \frac{p_{1}-p_{2}}{D_{p 1 p 2}}\right| .
$$

It's easy to calculate the distance $D$ between two pixels. The distance $D$ between two adjacent pixels is based on the geographic length. We define the distance from a center pixel to an adjacent pixel in its 4-neighborhood is 1 while the distance from this center pixel to an adjacent pixel in its 8-neighborhood except the pixel in its 4-neighborhood is $\sqrt{2}$.

Therefore we get the universal expression to calculate Dev of the route $i \mathrm{p} 0-\mathrm{p} 1 i-(\mathrm{p} 2 j$, $j \in[1,3]$ ) as follows:

$$
\operatorname{Dev}_{i}=\left|\begin{array}{l}
\arctan \frac{p_{0}-p_{1 i}}{D_{p 0 p 1 i}}- \\
\sum_{j} \arctan \left(\frac{p_{1 i}-p_{2 j}}{D_{p 1 i p 2 j}}\right) \\
j
\end{array}\right|, j \in[1,3],
$$

where $j$ is the number of pixels in the periphery on the route $i$. If $\mathrm{p}_{l i}$ has not the adjacent pixels in the periphery on the route $i$, that is, $j$ is zero, Dev of the route $i$ is unavailable.

Based on the definition of Dev, we can compare Dev with a threshold to distinguish whether a noise pixel candidate is an impulse noise pixel. However, if the noise intensity is high, some of neighbors are also noise pixel probably. Therefore we use some less Dev values on all routes for the noise detection. DSA is stated as follows.

\section{Algorithm 1 DSA}

\section{Stage 1}

Step 1) Convert original image to gray-level image with 8 bit. If the original image is a gray-level image with 8 bit, this step is omitted.

Step 2) Detect all pixel values that equal to 0 or 255 in this gray-level image and mark them as noise candidate, and then go to stage 2.

\section{Stage 2}

For every noise pixel candidate,

Step 1) Calculation of all available Dev for every route.

Step 2) Sort all Dev.

Step 3) Calculation of average on half of all smallest Dev. 
Step 4) Comparison between mean value and presetting threshold.

Step 5) Mark as impulse noise if average is larger than threshold.

\section{Experiments}

In order to test the performance of DSA, some experiments are tested. We use Microsoft Windows 10 as operation system and Matlab R2009b as the test platform. Two experiments are studied for the performance analysis and comparison. The first experiment is to analyze the performance of DSA, while the second experiment is for the comparison with other stateof-the-art methods.

First experiment is to test the fundamental performance of DSA. Table 1 shows the detection result of Lena under the different noise intensity. The results demonstrate DSA is robust under the different noise intensity, especially in the circumstance of high noise intensity. Table 2 shows the detection details of pepper (0) and salt (255) for Lena under the different noise intensity. The same to Table 1, DSA is also very stable to detect pepper noise pixels and salt noise pixels efficiently in the different circumstance.

Table 1. Detection result of DSA for Lena under the different noise intensity (Threshold is $\pi / 12$ ).

\begin{tabular}{cccc}
\hline Noise Percent & $\begin{array}{c}\text { Noise Candidate in } \\
\text { Polluted Image }\end{array}$ & $\begin{array}{c}\text { Detected Noise in } \\
\text { Polluted Image }\end{array}$ & Accuracy \\
\hline $10 \%$ & 26182 & 24916 & $95.16 \%$ \\
$20 \%$ & 52339 & 50733 & $96.93 \%$ \\
$30 \%$ & 78265 & 76813 & $98.14 \%$ \\
$40 \%$ & 104766 & 103601 & $98.89 \%$ \\
$50 \%$ & 130975 & 130053 & $99.30 \%$ \\
$60 \%$ & 157214 & 156473 & $99.53 \%$ \\
$70 \%$ & 183234 & 182369 & $99.53 \%$ \\
$80 \%$ & 209522 & 207559 & $99.06 \%$ \\
$90 \%$ & 235972 & 230878 & $97.84 \%$ \\
$95 \%$ & 249012 & 240520 & $96.59 \%$ \\
\hline
\end{tabular}

Table 2. Pepper (0) and salt (255) detection details of DSA for Lena under the different noise intensity (Threshold is $\pi / 12$ ).

\begin{tabular}{ccccccc}
\hline $\begin{array}{c}\text { Noise } \\
\text { percent }\end{array}$ & $\begin{array}{c}\text { Pepper (0) } \\
\text { candidate } \\
\text { in polluted } \\
\text { image }\end{array}$ & $\begin{array}{c}\text { Detected } \\
\text { Pepper } \\
\text { noise }\end{array}$ & $\begin{array}{c}\text { Pepper } \\
\text { Detected } \\
\text { Accuracy } \\
\text { Percent }\end{array}$ & $\begin{array}{c}\text { Salt (255) } \\
\text { candidate } \\
\text { in polluted } \\
\text { image }\end{array}$ & $\begin{array}{c}\text { Detected } \\
\text { Salt } \\
\text { Noise }\end{array}$ & $\begin{array}{c}\text { Salt } \\
\text { Detected } \\
\text { Accuracy } \\
\text { Percent }\end{array}$ \\
\hline $10 \%$ & 13002 & 12360 & $95.06 \%$ & 13180 & 12556 & $95.27 \%$ \\
$20 \%$ & 26094 & 25261 & $96.81 \%$ & 26245 & 25472 & $97.05 \%$ \\
$30 \%$ & 39149 & 38383 & $98.04 \%$ & 39116 & 38430 & $98.25 \%$ \\
$40 \%$ & 52349 & 51769 & $98.89 \%$ & 52417 & 51832 & $98.88 \%$ \\
$50 \%$ & 65675 & 65199 & $99.28 \%$ & 65300 & 64854 & $99.32 \%$ \\
$60 \%$ & 78227 & 77850 & $99.52 \%$ & 78987 & 78623 & $99.54 \%$ \\
$70 \%$ & 91656 & 91215 & $99.52 \%$ & 91578 & 91154 & $99.54 \%$ \\
$80 \%$ & 104900 & 103866 & $99.01 \%$ & 104622 & 103693 & $99.11 \%$ \\
$90 \%$ & 118220 & 115564 & $97.75 \%$ & 117752 & 115314 & $97.93 \%$ \\
$95 \%$ & 124094 & 120153 & $96.82 \%$ & 124918 & 120357 & $96.36 \%$ \\
\hline
\end{tabular}


Table 3. Comparison of noise detection results for lena with fixed-valued impulse noise.

\begin{tabular}{ccccccccc}
\hline $\begin{array}{c}\text { Noise } \\
\text { percent }\end{array}$ & \multicolumn{2}{c}{ SBF[4] } & \multicolumn{2}{c}{ ROR-NLM[5] } & \multicolumn{2}{c}{ ABDND[7] } & \multicolumn{2}{c}{ DSA } \\
& $\begin{array}{c}\text { False- } \\
\text { Hit }\end{array}$ & Miss-Hit & False-Hit & Miss-Hit & $\begin{array}{c}\text { False- } \\
\text { Hit }\end{array}$ & $\begin{array}{c}\text { Miss- } \\
\text { Hit }\end{array}$ & $\begin{array}{c}\text { False- } \\
\text { Hit }\end{array}$ & $\begin{array}{c}\text { Miss- } \\
\text { Hit }\end{array}$ \\
\hline $10 \%$ & 0 & 36 & 834 & 1 & 7 & 0 & 0 & 1266 \\
$20 \%$ & 0 & 45 & 731 & 10 & 0 & 0 & 0 & 1606 \\
$30 \%$ & 0 & 112 & 726 & 63 & 0 & 0 & 0 & 1452 \\
$40 \%$ & 0 & 717 & 808 & 577 & 0 & 0 & 0 & 1165 \\
$50 \%$ & 0 & 3122 & 1443 & 2280 & 0 & 0 & 0 & 922 \\
$60 \%$ & 0 & 10949 & 3441 & 7335 & 0 & 0 & 0 & 741 \\
$70 \%$ & 0 & 29606 & 7393 & 20188 & 0 & 0 & 0 & 865 \\
$80 \%$ & 0 & 62121 & 12468 & 48260 & 0 & 0 & 0 & 5094 \\
$90 \%$ & 0 & 111371 & 12698 & 92962 & 0 & 0 & 0 & 8492 \\
\hline
\end{tabular}

In the second experiment we compare the performance between DSA and other 3 methods. Table 3 illustrates the noise detection results of different methods for Lena with the different fixed-valued impulse noise. From this table, we know both the false-hit number (rate) and the miss-hit number (rate) keep in a low level in not only the different image detection but also the different noise intensity. In contrast to DSA, ROR-NLM has not stable performance for different images. SBF is an efficient method when the noise intensity is low (less than $0.5)$ but has bad performance when the noise intensity is high. ABDND is an excellent method with significant miss-hit rate. But most original pixels whose values are 0 or 255 are detected as the noise pixel in ABDND detection. The performance of DSA is not the most excellent in most of occasions. However, the performance of DSA is very stable in the different image detection and the different noise intensity (from 0 to 0.95 ). Moreover, when the noise intensity is high, the running times of ABDND and ROR-NLM are very long but the running times of SBF and DSA are short. If the lookup table method is used to calculate the inverse trigonometric function in DSA implementation, the running times of DSA will be shorter so that DSA can be used in the field of real-time application.

\section{Conclusions}

The main contribution of this paper is to propose a new method to detect the fixed-valued impulse noise. Distinguishing from the traditional average methods and medium methods that are widely studied, this method uses the deviation of slant angle for every pixel candidate as the criterion for noise detection. The most advantage of this method is to detect the fixedvalued impulse noise effectively in the circumstance of very high noise intensity (up to 0.95 ). The experiment results verify this method has good performance under the different noise intensity. This method uses the deviation of slant angle as the test object so that some normal pixels in the edge perhaps are detected as noise pixel. When the method is used for the detection of random-valued impulse noise, the false-hit number should be larger because many normal pixels in the edge are recognized as noise pixel. Therefore this method needs to add some additional criterions for detection of random-valued impulse noise. In a word, this method is a useful method for the detection of fixed-valued impulse noise, especially under the noise pollution with very high noise intensity. The mathematical model, the detection algorithm and the detection object of this method will be studied to expand the application area of this method in the future work.

The generous support of the Discipline Construction Fund of SSPU (XXKZD1605) and the Construction of University Enterprise Cooperation Automobile Electronic Joint Experiment Center (A11NH182016) are gratefully acknowledged. 


\section{Reference}

1. R. C. Gonzalez and R. E. Woods, Digital Image Processing. Englewood Cliffs, NJ: Prentice-Hall, 2002.

2. T. Chen, K. K. Ma, and L. H. Chen, “Tri-state median filter for image denoising," IEEE Trans. Image Process., vol. 8, no. 12, pp. 1834-1838, Dec. 1999.

3. T. Chen and H. R. Wu, "Space variant median filters for the restoration of impulse noise corrupted images,” IEEE Trans. Circuits Syst. II, Analog Digit. Signal Process., vol. 48, no. 8, pp. 784-789, Aug. 2001.

4. C. Lin, J. Tsai, and C. Chiu, "Switching bilateral filter with a texture/noise detector for universal noise removal," IEEE Trans. Image Process., vol. 19, no. 9, pp. 2307-2320, Sep. 2010.

5. X. Bo and Z. Yin, "A universal denoising framework with a new impulse detector and nonlocal means," IEEE Trans. Image Process., vol. 21, no. 4, pp. 1663-1675, Apr. 2012.

6. X. Zhang and Y. Xiong, "Impulse noise removal using directional difference based noise detector and adaptive weighted mean filter", IEEE Signal Process.Lett., vol. 16, no. 4, pp.295-298, Apr. 2009.

7. A. K. Tripathi, U. Ghanekar, and S. Makhopadhyay, "Switching median filter: advanced boundarydiscriminative noise detection algorithm," IET Image Process., Vol. 5, no. 7, pp. 598-610, Jul, 2011. 